\title{
Investigation of nickel lattice sites in diamond: Density functional theory and x-ray absorption near-edge structure experiments
}

\author{
Etienne Gheeraert, ${ }^{*}$ Amit Kumar, ${ }^{\dagger}$ Etienne Bustarret, Laurent Ranno, Laurence Magaud, and Yves Joly \\ Institut Néel, CNRS and Université Joseph Fourier, 25 rue des Martyrs, BP166, 38042 Grenoble Cedex 9, France \\ Sakura Pascarelli and Matthew Ruffoni \\ European Synchrotron Radiation Facility (ESRF), Boîte Postale 220, F-38043 Grenoble Cedex, France \\ Devesh Kumar Avasthi
Materials Science Group, Inter-University Accelerator Centre, New Delhi 110067, India
}

Hisao Kanda ${ }^{\ddagger}$

National Institute for Materials Science, 1-1 Namiki, 305-0044 Tsukuba, Japan

(Received 4 May 2012; revised manuscript received 30 July 2012; published 22 August 2012)

\begin{abstract}
Possible lattice incorporation sites for $\mathrm{Ni}$ in diamond have been investigated using ab initio density functional theoretical calculations. The results have been used to compute x-ray absorption near-edge structure spectra which were compared to spectroscopic measurements performed on a diamond single crystal grown at high pressure and high temperature in a nickel solvent. $\mathrm{Ni}$ at divacancy sites is proposed to be the most stable and probable configuration in this crystal.
\end{abstract}

DOI: 10.1103/PhysRevB.86.054116

PACS number(s): 61.72.Bb, 61.72.up, 61.72.Dd, 71.15.Nc

\section{INTRODUCTION}

Besides purely academic issues, interest in the so-called color centers of diamond, nurtured for decades by esthetical and economical gemnology-related stakes, has recently been renewed by the possible application of such centers to quantum information processing. The weak coupling of impurities with lattice phonons of this transparent crystal, combined with its high thermal conductivity, leads to defects which may be investigated at an individual level ${ }^{1}$ and can act as highly photostable single photon sources operating at room temperature. The most striking example is the nitrogen-vacancy (NV) center which has been used as both an electrically controllable single photon source $^{2}$ and a nanoscale magnetometer, ${ }^{3,4}$ but other impurities such as $\mathrm{Si}^{5,6}$ or transition metals ${ }^{7-12}$ are also currently investigated for their optical emission quantum properties. Ion implantation has been quite popular in this context, ${ }^{12,13}$ but a few impurities may also be incorporated within the dense diamond crystal during chemical vapor deposition or high pressure and high temperature (HPHT) growth without clustering: on substitutional sites, $\mathrm{B}$ (electron acceptor), $\mathrm{P}$ (donor), $\mathrm{N}$, and $\mathrm{Si}$; and as intersitials, $\mathrm{H}, \mathrm{He}$, and $\mathrm{Li}$. To our knowledge, the only transition metals in this category are $\mathrm{Co}^{8}$ and $\mathrm{Ni}^{7,14} \mathrm{Co}$ has been tentatively suggested as a dopant in diamond seen as a new diluted magnetic semiconductor for spintronics. ${ }^{15-17} \mathrm{Ni}$ in diamond has been frequently proposed as a single photon source for quantum cryptography, ${ }^{10-13,18}$ as well as a deep trap for radiation dosimetry ${ }^{19,20}$ or a pulsed magnetic field calibration probe ${ }^{21}$ Contrary to $\mathrm{B}, \mathrm{P}$, and $\mathrm{N}$ for which extensive physicochemical data are available, equivalent experimental knowledge about $\mathrm{Co}$ - or $\mathrm{Ni}$-doped diamond is severely lacking. One of the objectives of the present $\mathrm{x}$-ray absorption near-edge structure (XANES) study at the K edge of $\mathrm{Ni}$ is to partially bridge this gap.

Over the last two decades, several theoretical approaches have been applied to Ni-related defects in diamond, using first small cluster calculations where atomic relaxation processes were not described properly. ${ }^{22-27}$ The subject was then revisited employing $a b$ initio density functional theory (DFT) calculations with periodic boundary conditions, ${ }^{28-30}$ providing additional insight into the microscopic structure of various Ni-related centers in diamond. A general conclusion of the more recent studies is that isolated $\mathrm{Ni}$ atoms, either substitutional or at one of the interstitial sites, are not so stable and that complexes of one $\mathrm{Ni}$ atom with vacancies or other chemical impurities are more likely to occur under equilibrium conditions. However, not all open issues have been settled. In this work we not only calculate by $a b$ initio methods the energy and stability of the Ni lattice sites but also compare quantitatively the experimental data to the simulated XANES response computed for each relaxed site.

From an experimental point of view, the various lines detected by electronic paramagnetic resonance (EPR) ${ }^{31-36}$ or by magneto-optical (including magnetic circular dichroism) or piezo-optical spectroscopy ${ }^{21,37-39}$ have been attributed to specific incorporation sites of $\mathrm{Ni}$ in diamond. This has been a challenge since the early days when it was recognized that an isolated $\mathrm{Ni}$ atom was incorporated in a site of trigonal symmetry at low temperatures (below $25 \mathrm{~K}$ ) changing over to a tetrahedral symmetry at higher temperatures. Twenty years later, despite the efforts of both experimentalists and theoreticians, the identification of $\mathrm{Ni}$ lattice sites in diamond remains controversial. ${ }^{30,35}$ Our contribution to this debate is based on the characterization of one nickel-doped diamond crystal by cathodoluminescence, magnetometry, and XANES presented in Sec. III, on the DFT calculations reported in Sec. IV, and on the theoretical XANES spectra described in Sec. V. The microscopic nature of the Ni lattice sites in this sample are discussed in Sec. VI. In this section, before concluding, we also tentatively relate the present analysis to the results of a thorough magneto-optical study performed recently at high fields and low temperatures on the same diamond crystal. $^{39}$ 


\section{TECHNICAL DETAILS}

The diamond crystal studied was grown in pure nickel solvent under HPHT conditions and has a yellow color without any visible inclusion. Because no nitrogen getter was added to the solvent, a significant nitrogen contamination occurred in the crystal. The type Ib diamond seed crystal was removed before characterization. According to secondary ion mass spectroscopy measurements, performed on a different sample grown under similar conditions, the nickel concentration is expected to be around $10^{19} \mathrm{~cm}^{-3}$, and the nitrogen concentration is expected to be about the same. Note that due to the dependence of the impurity incorporation rate on the crystallographic orientation during growth, the impurity concentration varies depending on the growth sector. The crystal was characterized by cathodoluminescence at $5 \mathrm{~K}$ with a $30-\mathrm{kV}$ electron beam. Magnetic properties were measured using a superconducting quantum interference device (SQUID) from $2 \mathrm{~K}$ to $300 \mathrm{~K}$. $\mathrm{X}$-ray absorption near edge structure (XANES) experiments are performed at the beam line BM29 at the European Synchrotron Radiation Facility, Grenoble. Spectra were measured at the Ni K edge $\left(E_{0}=8.3 \mathrm{keV}\right)$ in fluorescence mode, using a $\mathrm{Ge}$ solid state detector oriented at $45^{\circ}$ with respect to $\mathrm{x}$-ray propagation direction.

The structure and electronic properties of nickel and nickel-nitrogen complexes were investigated using the Vienna Ab-initio Simulation Package (VASP) code. ${ }^{40}$ It is based on density functional theory (DFT) within the generalized gradient approximation. ${ }^{41}$ A projector augmented wave was used with a basis cutoff equal to $368 \mathrm{eV}^{42}$ The Monkhorst-Pack scheme ${ }^{43}$ was used for integration in the Brillouin zone on a $5 \times 5 \times 5$ $k$-point mesh. The calculations were performed in a $3 \times 3 \times 3$ cubic cell (216 atoms). After convergence, residual forces were lower than $0.012 \mathrm{eV} / \AA$. The calculation of the energy band gap for pure diamond $\left(E_{g}=4.2 \mathrm{eV}\right)$ is in good agreement with theoretical calculations. ${ }^{44,45}$ Total energies come out of the calculations but a direct comparison is not possible when the complexes involve different numbers of atoms of different kinds. To do so, we calculated the grand potential $\Omega(X)$ for the different complexes $X$ in a neutral charge state:

$$
\Omega(X)=E(X)-\sum_{i} \mu_{i} n_{i},
$$

where $E(X)$ is the total energy of the supercell that models complex $X, n_{i}$ is the number of atoms of species $i$, and $\mu_{i}$ is their chemical potential. The chemical potential of carbon atoms was deduced from the total energy of a $3 \times 3 \times 3$ diamond cell, $\mu_{C}=E$ (diamond)/216. We made no assumption for the chemical potentials of $\mathrm{Ni}$ and $\mathrm{N}$. As a consequence, we can compare the total energies of $\mathrm{Ni}$ centers that involve different numbers of $\mathrm{C}$ atoms as long as the relative numbers of $\mathrm{Ni}$ and $\mathrm{N}$ atoms remain the same. For each class of complex (class $1, \mathrm{C}$ atoms $+\mathrm{Ni}$ atoms; class $2, \mathrm{C}$ atoms $+1 \mathrm{Ni}+1 \mathrm{~N}$; class 3, C atoms $+1 \mathrm{Ni}+2 \mathrm{~N}$ ), we calculated corrected energies according to Eq. (1). This corrected energy is the one to be used to discuss relative stability.

Experimental XANES spectra were simulated using the FDMNES code. ${ }^{46}$ This code is also $a b$ initio DFT but uses the multiple scattering theory (MST) in a cluster approach, well adapted for the photoelectron energy range. It has been checked that working with the finite difference method, allowing a more precise description of the potential, did not improved the results. The X-ray absorption spectra were calculated for all the possible sites listed in Table I. As input we used the relaxed atomic positions obtained by the DFT

TABLE I. Summary of total energy and magnetic moment of Ni defects in a diamond matrix. The corrected energy is the energy calculated using Eq. (1) for a common reference.

\begin{tabular}{|c|c|c|c|c|c|}
\hline Lattice site & Name & Cluster composition & $\begin{array}{l}\text { Total energy } \\
(\mathrm{eV})\end{array}$ & $\begin{array}{l}\text { Corrected energy } \\
(\mathrm{eV})\end{array}$ & $\begin{array}{c}\text { Magnetic moment } \\
\left(\mu_{B}\right)\end{array}$ \\
\hline Diamond & Diamond & $216 \mathrm{C}$ & -1961.24 & 0 & 0.0 \\
\hline Class 1: $1 \mathrm{Ni}$ & Corrected energies per $\mathrm{Ni}$ atom & & & & \\
\hline $\mathrm{Ni}_{S}$ & Substitutional & $215 \mathrm{C}+1 \mathrm{Ni}$ & -1950.358 & 1.799 & 1.97 \\
\hline $\mathrm{Ni}_{T}$ & Tetrahedral interstitial & $216 \mathrm{C}+1 \mathrm{Ni}$ & -1952.740 & 8.5 & 0.2 \\
\hline $\mathrm{Ni}_{H}$ & Hexagonal interstitial & $216 \mathrm{C}+1 \mathrm{Ni}$ & -1952.399 & 8.837 & 0.2 \\
\hline $\mathrm{Ni}_{S} \mathrm{Ni}_{S}$ & Ni pair ${ }^{\mathrm{a}}$ & $214 \mathrm{C}+2 \mathrm{Ni}$ & -1941.67 & & \\
\hline $\mathrm{Ni}_{S}-\mathrm{Ni}_{S}$ & Two distant $\mathrm{Ni}^{\mathrm{b}}$ & $214 \mathrm{C}+2 \mathrm{Ni}$ & -1938.95 & & 3.97 \\
\hline $\mathrm{VNi}_{I} \mathrm{~V}$ & $\mathrm{Ni}_{I}$ divacancy & $214 \mathrm{C}+1 \mathrm{Ni}$ & -1942.977 & 0.1 & 2.14 \\
\hline Class 2: $1 \mathrm{Ni}, 1 \mathrm{~N}$ & Corrected energies per $(\mathrm{Ni}, \mathrm{N})$ pair & & & & \\
\hline $\mathrm{Ni}_{S} \mathrm{~N}_{S}$ & $\mathrm{Ni}-\mathrm{N}$ complex & $214 \mathrm{C}+1 \mathrm{Ni}+1 \mathrm{~N}$ & -1949.619 & -6.54 & 1.0 \\
\hline $\mathrm{Ni}_{T} \mathrm{~N}_{S}$ & $\mathrm{Ni}-\mathrm{N}$ complex & $215 \mathrm{C}+1 \mathrm{Ni}+1 \mathrm{~N}$ & -1949.81 & 2.35 & \\
\hline $\mathrm{Ni}_{S} \mathrm{~N}_{T}$ & $\mathrm{Ni}-\mathrm{N}$ complex & $215 \mathrm{C}+1 \mathrm{Ni}+1 \mathrm{~N}$ & -1947.35 & 4.81 & \\
\hline $\mathrm{N}_{S} \mathrm{VNi}_{I} \mathrm{~V}$ & $\mathrm{Ni}$ divacancy $+1 \mathrm{~N}$ & $213 \mathrm{C}+1 \mathrm{Ni}+1 \mathrm{~N}$ & -1943.283 & -9.29 & 1.03 \\
\hline Class 3: $1 \mathrm{Ni}, 2 \mathrm{~N}$ & Corrected energies for $1 \mathrm{Ni}$ and $2 \mathrm{~N}$ & & & & \\
\hline $\mathrm{N}_{S} \mathrm{~N}_{S} \mathrm{VNi}_{i} \mathrm{~V}$ & $\mathrm{Ni}$ divacancy $+2 \mathrm{~N}$ & $212 \mathrm{C}+1 \mathrm{Ni}+2 \mathrm{~N}$ & -1943.214 & -18.30 & 0 \\
\hline $\mathrm{N}_{S} \mathrm{VNi}_{I} \mathrm{VN}_{S}$ & $\mathrm{Ni}$ divacancy $+2 \mathrm{~N}^{\mathrm{c}}$ & $212 \mathrm{C}+1 \mathrm{Ni}+2 \mathrm{~N}$ & -1942.546 & -17.63 & 0 \\
\hline $\mathrm{N}_{S} \mathrm{VNi}_{I} \mathrm{VN}_{S}$ & $\mathrm{Ni}$ divacancy $+2 \mathrm{~N}^{\mathrm{d}}$ & $212 \mathrm{C}+1 \mathrm{Ni}+2 \mathrm{~N}$ & -1943.034 & -18.12 & 0 \\
\hline
\end{tabular}

a Two substitutional Ni atoms as first neighbor (separation distance $a \frac{\sqrt{3}}{4}, a$ being the diamond lattice constant).

${ }^{\mathrm{b}}$ Two substitutional Ni atoms separated by one lattice constant $a$.

${ }^{\mathrm{c}}$ One $\mathrm{N}$ atom on each side of an $\mathrm{Ni}$ atom with inversion symmetry.

${ }^{\mathrm{d}}$ One $\mathrm{N}$ atom on each side of an $\mathrm{Ni}$ atom without inversion symmetry. 
calculation. We also used the nonrelaxed ones for comparison. Convergence versus the radius of the cluster around the absorbing atom was checked. At $5 \AA$ (that is, with an 85 -atom cluster), it is already satisfactory. The first series of simulations was thus performed using this radius in order to select the best $\mathrm{Ni}$ site. When this one was found, further refinements were performed with a $7-\AA$ radius, that is, with around 255 atoms in the cluster.

\section{DIAMOND CRYSTAL CHARACTERIZATION}

The diamond crystal was first characterized by cathodoluminescence. The spectrum (Fig. 1) recorded on growth sector (111), reveals mainly the so-called $1.4-\mathrm{eV}$ nickel center. The high spectral resolution spectrum reveals two peaks of similar intensities located at 1.401 and $1.404 \mathrm{eV}$. Careful optical studies of the fine structure attributed unambiguously this center to a defect containing one single nickel atom, ${ }^{47}$ the intensities of the lines observed in the fine structure being related to the natural abundances of nickel isotopes. This optical feature has been unambiguously associated with the EPR center NIRIM-2, ${ }^{47}$ but has a controversial origin. It has been attributed to isolated interstitial nickel $\mathrm{Ni}_{i}^{+},{ }^{29} \mathrm{VNi}_{i}^{+}\left(3 d^{9}\right)$ in a $\mathrm{NiB}$ complex, ${ }^{35}$ and recently to the nickel divacancy $\mathrm{VNi}_{i}^{+} \mathrm{V}$ (Ref. 36) (also called double semivacancy if $\mathrm{Ni}$ is considered in a substitutional site). Besides this dominant feature, the nickel-related 2.56-eV center and two nitrogenrelated complexes, at 2.463 and $2.156 \mathrm{eV}$, appear on growth sector (111). Note that the incorporation of nickel depends on the concentration of nitrogen. The 1.4-eV center is observed only in diamond crystals with low nitrogen concentration, typically below $5 \times 10^{19} \mathrm{~cm}^{-3}$. For nitrogen-rich crystals $([\mathrm{N}] \gg[\mathrm{Ni}])$, the $1.4-\mathrm{eV}$ center is weak and other optical structures such as 1.883 and $2.51 \mathrm{eV}$ are dominant. ${ }^{48}$ The main incorporation site of nickel is therefore expected to be the one associated with the 1.4-eV center as the nitrogen concentrate is low in the crystal studied, and the absence of the 793.6-nm line confirms that nitrogen impurities are not in aggregates. $^{49}$

Magnetometry measurements show at $300 \mathrm{~K}$ the diamagnetic component of the diamond crystal and at $4 \mathrm{~K}$ a paramagnetic contribution of the crystal (Fig. 2). Assuming a

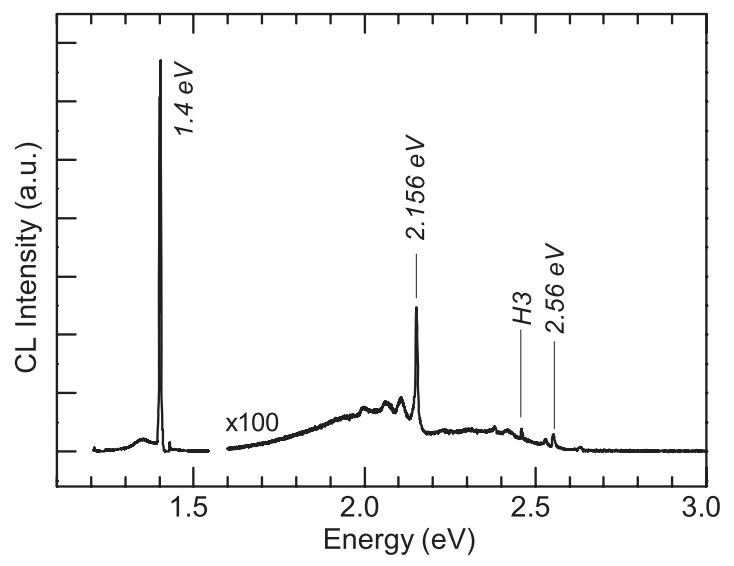

FIG. 1. Cathodoluminescence spectrum recorded at $5 \mathrm{~K}$ on the (111) growth sectors of the nickel-doped diamond crystal.

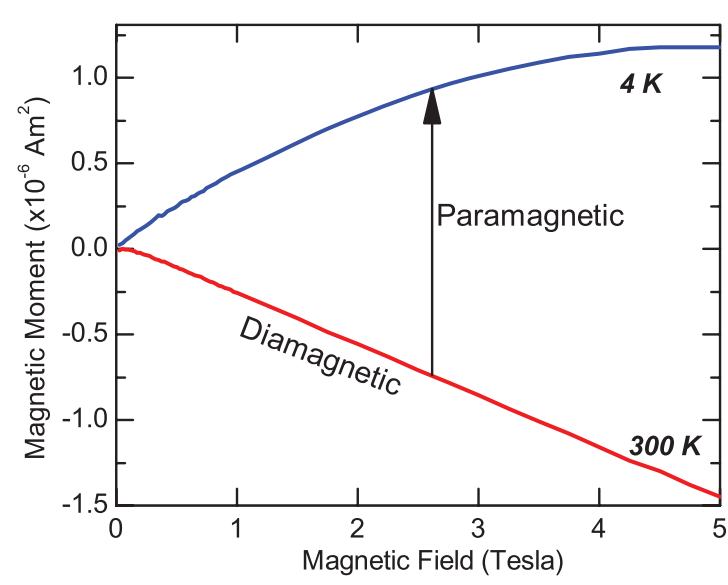

FIG. 2. (Color online) Magnetization of the Ni-doped diamond crystal recorded at 4 and $300 \mathrm{~K}$. The crystal has a diamagnetic behavior at $300 \mathrm{~K}$, with negative susceptibility, and paramagnetic behavior at $4 \mathrm{~K}$.

magnetic moment of $2 \mu_{B}$ per magnetic center $\left(\mu_{B}\right.$ being the Bohr magneton), as it would be for $\mathrm{Ni}_{S}$ and $\mathrm{VNi}_{I} \mathrm{~V}$ centers according to DFT calculations (see below), a concentration of $1.5 \times 10^{19} \mathrm{~cm}^{-3}$ centers was deduced. Such a Ni incorporation corresponds to a relative concentration of $80 \mathrm{ppm}$; i.e., the $\mathrm{Ni}-\mathrm{Ni}$ average first neighbor distance corresponds to 25 carbon atoms. In such a highly diluted magnetic system, weak interactions between magnetic ions and paramagnetic behavior are expected. This value being of the same order of magnitude as the nickel concentration, we conclude that a significant part of the nickel atoms are incorporated in noninteracting isolated magnetic centers. In particular, there is no significant nickel clustering in this crystal that would have induced a ferromagnetic component of the crystal magnetic moment. The Ni K edge X-ray absorption spectrum of the diamond crystal is shown in Fig. 3, with the absorption edge appearing at $8.33 \mathrm{keV}$, corresponding to electronic transitions from the $1 s$ core level. Near-edge structures, or XANES, induced by interference between the X-ray photoelectron and the local potential fluctuations, reveal the local environment of $\mathrm{Ni}$. Higher energy fine structures, or EXAFS, are not exploitable because of poor signal to noise ratio. As a first comparison,

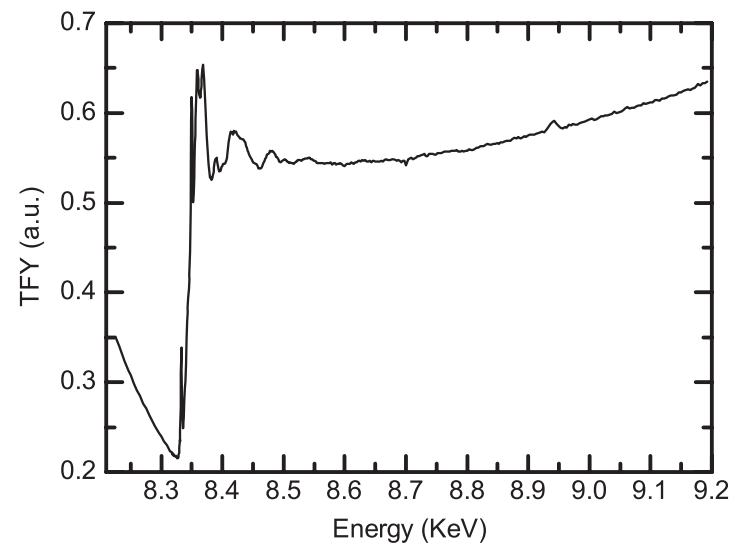

FIG. 3. XANES spectrum of the Ni-doped diamond crystal recorded near the $\mathrm{Ni} \mathrm{K}$ absorption edge. 


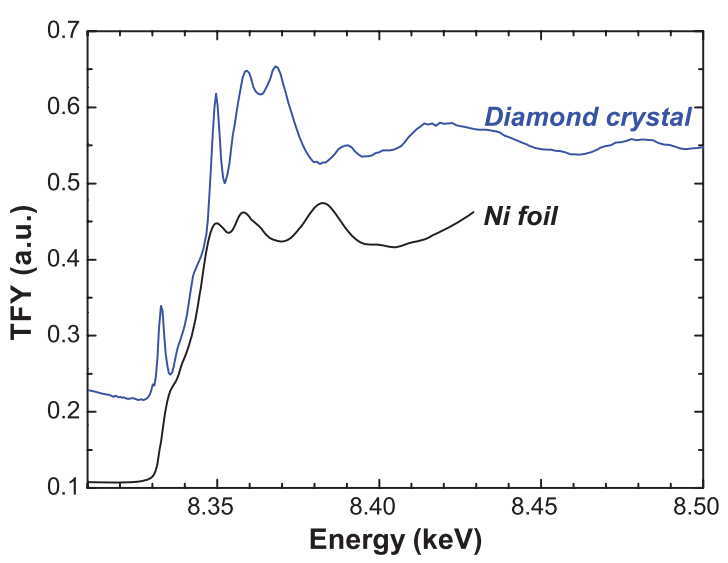

FIG. 4. (Color online) X-ray absorption of the Ni-doped diamond crystal compared to the one on a nickel foil.

a XANES signal of a nickel foil recorded in transmission is also plotted in Fig. 4. If the threshold appears at the same energy, the detailed fine structure indicates that the lattice site of $\mathrm{Ni}$ in diamond is obviously different from that in bulk nickel.

\section{DFT CALCULATIONS}

The most probable $\mathrm{Ni}$ lattice sites in diamond were analyzed by DFT calculations. Results are gathered in Table I. We determined total energies, relaxed atomic positions, and magnetic moments for different classes of defects: the first category only involves $\mathrm{Ni}$ atoms; the second class one $\mathrm{Ni}$ atom and one $\mathrm{N}$ atom; and in the third class, the $\mathrm{N}$ density is twice the Ni density. We start with the first class of defects. The substitutional site $(\mathrm{S})$ is obtained by replacing one $\mathrm{C}$ atom by one $\mathrm{Ni}$ atom, as shown in Fig. 5. The tetrahedral (T) interstitial site lies equidistant from four carbon atoms and the hexagonal $(\mathrm{H})$ interstitial site lies midway between two $\mathrm{T}$ sites. In this first case, we correct the calculated total energy of the different cells by subtracting the number of $\mathrm{C}$ atoms times $\mu_{C}$ and dividing by the number of $\mathrm{Ni}$ atoms. As already pointed by Goss et al. ${ }^{28}$ the substitutional site is the most stable configuration within these three complexes. The $\mathrm{Ni}_{S}$ atom carries a magnetic moment equal to $1.97 \mu_{B} . \mathrm{H}$ and $\mathrm{T}$ sites have much smaller magnetic moments $\left(\mu=0.2 \mu_{B}\right)$. The $\mathrm{Ni}_{S}$ relaxed atomic position is shown in Fig. 5. The Ni atom induces an outward relaxation of the adjacent carbon atoms. This occurs because the radius of $\mathrm{Ni}$ is $57 \%$ larger than that of carbon. The Ni-C first nearest-neighbor distance is equal to $1.77 \AA$ (C-C equal to $1.54 \AA$ in diamond). To mimic $\mathrm{Ni}$ segregation, we then modeled substitutional $\mathrm{Ni}$ pairs in the first neighbor sites separated by a diamond lattice vector. As shown in Table I the first neighbor Ni pair is the most stable one.

Finally, other low energy sites for transition metals in silicon $^{50}$ and diamond ${ }^{34,51}$ have been proposed, in particular, the double semivacancy $\mathrm{V}_{1 / 2} \mathrm{Ni}_{S} \mathrm{~V}_{1 / 2}$, also called divacancy $\mathrm{VNiV}$. A divacancy site is created by removing two neighboring $\mathrm{C}$ atoms and placing one $\mathrm{Ni}$ at their midpoint, as shown in Fig. 5 in relaxed position. Among all the lattice sites that only involve $\mathrm{Ni}$ atoms, the $\mathrm{Ni}$ divacancy is the energetically

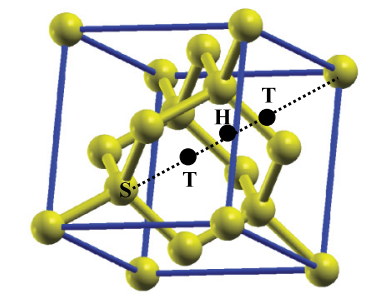

(a)

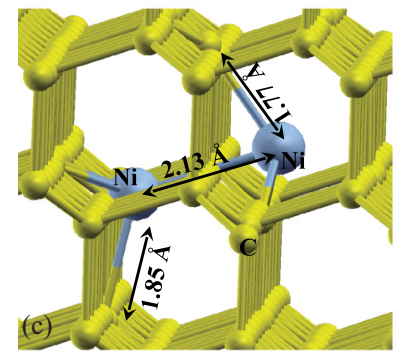

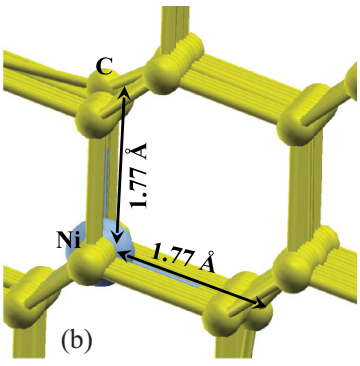

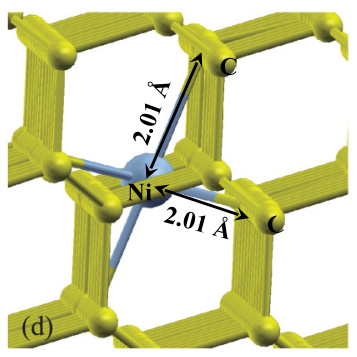

FIG. 5. (Color online) (a) Schematic of the possible sites for foreign atoms in diamond cell. The substitutional site (S) replacing one carbon atom by foreign atoms. The tetrahedral $(\mathrm{T})$ sites lie equidistant from four carbon atoms and the hexagonal $(\mathrm{H})$ site lies midway between two $\mathrm{T}$ sites. Completely relaxed structure of a $\mathrm{Ni}$ atom at (b) substitutional $\mathrm{Ni}_{S}$, (c) two nearest-neighbor $\mathrm{Ni}_{S}$ atoms, and $(\mathrm{d}) \mathrm{Ni}$ divacancy $\left(\mathrm{VNi}_{I} \mathrm{~V}\right)$. The carbon atoms are shown as golden circles and the nickel atoms are shown as blue circles.

most favorable configuration. The $\mathrm{Ni}$ atom carrying a magnetic moment equal to $2.1 \mu_{B}$.

In order to understand the effect of nitrogen on the incorporation of nickel, $\mathrm{Ni}-\mathrm{N}$ complexes have been studied in the following configurations (corresponding to the second group of complexes) (Fig. 6):

(a) $\left(\mathrm{Ni}_{S}-\mathrm{N}_{S}\right)$ complex, both nickel and nitrogen atoms at the substitutional sites;

(b) $\left(\mathrm{N}_{S}-\mathrm{Ni}_{T}\right)$ complex, nitrogen atom at the substitutional site and $\mathrm{Ni}$ atom at the tetrahedral site;

(c) $\left(\mathrm{Ni}_{S}-\mathrm{N}_{T}\right)$ complex, nickel atom at the substitutional site and nitrogen atom at the tetrahedral site; and

(d) $\left(\mathrm{VNi}_{I} \mathrm{~V}+\mathrm{N}_{S}\right)$ complex, $\mathrm{Ni}$ divacancy plus one $\mathrm{N}$ atom.

The corrected energies of Table I are expressed for one $(\mathrm{Ni}, \mathrm{N})$ pair.

In the case of the $\mathrm{Ni}-\mathrm{N}$ complex, $\left(\mathrm{Ni}_{S}-\mathrm{N}_{S}\right)$ is the most stable configuration of the three first complexes but the nitrogendecorated nickel divacancy where one $\mathrm{C}$ first neighbor is replaced by one $\mathrm{N}$ atom is even more stable. When nickel and nitrogen atoms lie at substitutional sites the $\mathrm{Ni}-\mathrm{N}$ bond length is $1.78 \AA$.

The last class of calculated defects involves a divacancy decorated by two $\mathrm{N}$ atoms (Fig. 7). Three configurations are possible: in the first one, the two $\mathrm{N}$ atoms are on the same side of the $\mathrm{Ni}$ atom. In the second and third configurations they are situated on both sides of the $\mathrm{Ni}$ atom with an inversion symmetry in the second case and none in the third. The case with two $\mathrm{N}$ atoms on the same side corresponds to the lowest energy. 


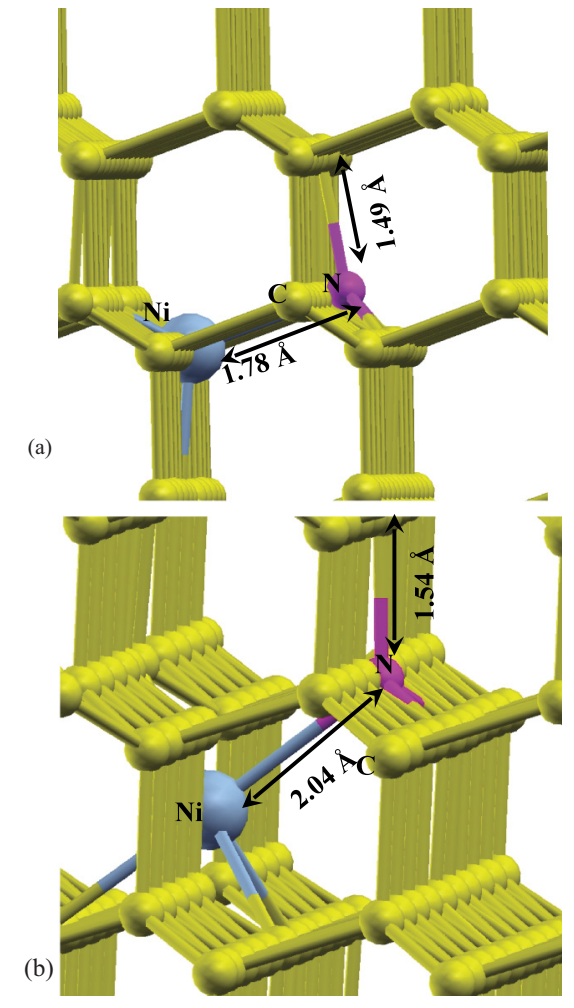

FIG. 6. (Color online) Relaxed structure of two configurations of the Ni-N complex: (a) $\left(\mathrm{Ni}_{S}-\mathrm{N}_{S}\right)$ complex, both nickel and nitrogen atoms at substitutional sites, and (b) Ni divacancy decorated with one substitutional nitrogen atom $\left(\mathrm{VNi}_{I} \mathrm{VN}_{S}\right)$

\section{XANES CALCULATIONS}

Figure 8 shows the XANES simulated spectra corresponding to the substitutional site $(\mathrm{S})$, the hexagonal interstitial site $(\mathrm{H})$, and the tetrahedral interstitial site $(\mathrm{T})$ for the $\mathrm{Ni}$ atom in diamond. When comparing with the experimental spectra, the $\mathrm{S}$ site could appear as the best because all the main features are present. Nevertheless their relative intensity is not well suited. The $\mathrm{T}$ site is definitely inadequate and can be rejected. The $\mathrm{H}$ site shows the double bump at $8.36-8.37 \mathrm{keV}$ but reveals also extra features on the rising edge. In the case of the $\mathrm{Ni}$ divacancy, the agreement is much better, most of the main features measured in the experiment are also present in the simulation with relatively well-matched intensities (Fig. 9). In the higher energy part some extra damping reveals probably a slightly disordered structure. In the same figure is shown $\left(\mathrm{NV}_{S} \mathrm{Ni}_{I} \mathrm{VN}_{S}\right)$ the nickel divacancy with two surrounding nitrogen atoms with inversion symmetry. In both models, the atomic positions are exactly the same, the only difference being that two carbon atoms are substituted by nitrogen atoms. It can be checked that the effect of the $\mathrm{N}$ atoms is rather small. There is only a slight improvement for the amplitude of the pre-edge peak at $8333 \mathrm{eV}$. When using relaxed structures, the difference between both models becomes more important. This difference comes thus from the atomic displacements induced by the $\mathrm{N}$ atoms and not directly from its chemical nature (the photoelectron scattering effects of $\mathrm{N}$ and $\mathrm{C}$ atoms are similar). Surprisingly the nonrelaxed divacancy model (shown in the

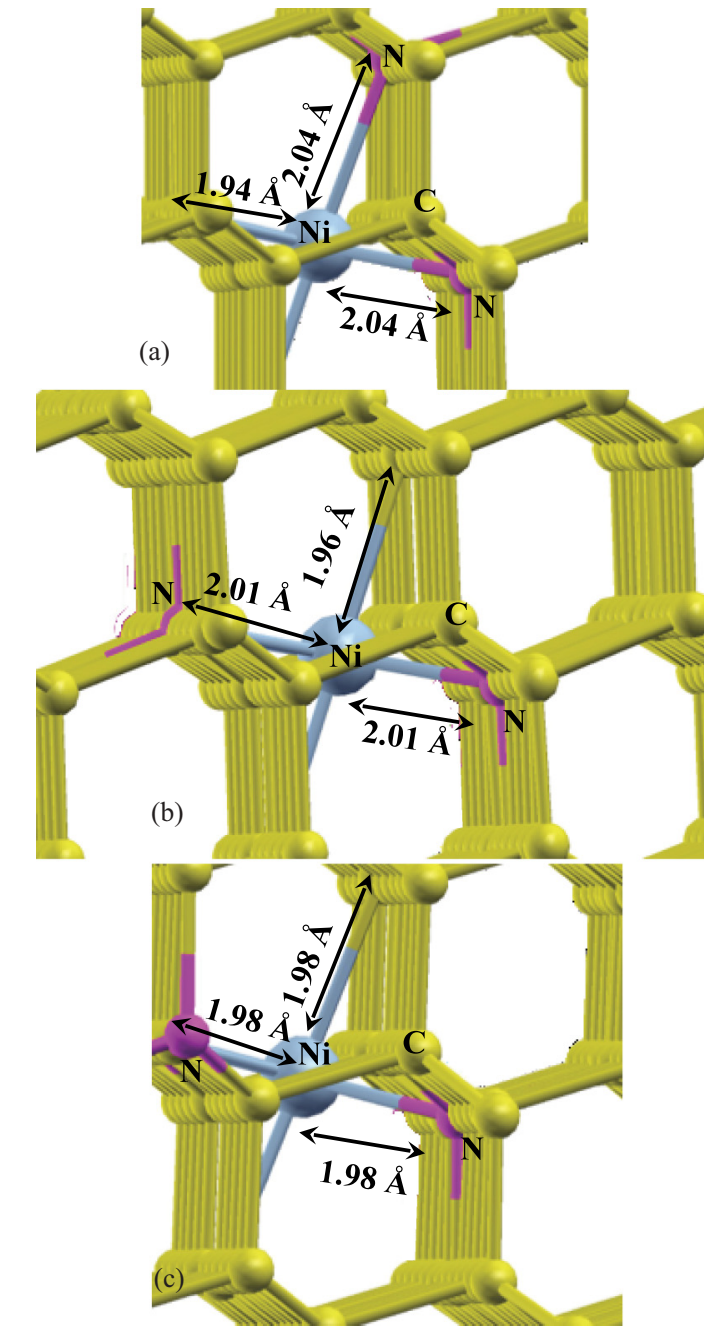

FIG. 7. (Color online) Relaxed structure of the three configurations of the Ni divacancy decorated with two substitutional nitrogen atoms: (a) $\left(\mathrm{VNi}_{I} \mathrm{VN}_{S} \mathrm{~N}_{S}\right)$ two $\mathrm{N}$ atoms on the same side of the $\mathrm{Ni}$ atom, (b) $\left(\mathrm{N}_{S} \mathrm{VNi}_{I} \mathrm{VN}_{S}\right)$ one $\mathrm{N}$ atom on each side of the $\mathrm{Ni}$ atom with inversion symmetry, and (c) $\left(\mathrm{N}_{S} \mathrm{VNi}_{I} \mathrm{VN}_{S}\right)$ one $\mathrm{N}$ atom on each side of the $\mathrm{Ni}$ atom without inversion symmetry.

figure) is slightly better than the relaxed one. It must be said that there are different possibilities for the $\mathrm{N}$ sites around the $\mathrm{Ni}$ atoms. Some tests showed that it was not possible to discriminate among them. Most probably, these different sites are allowed and the resulting experimental spectrum is an average corresponding to the disorder evoked above. In any case, from the XANES analysis, the divacancy model is notably more likely than the other configurations.

\section{DISCUSSION}

We have searched for the most stable structures for isolated nickel, $\mathrm{Ni}-\mathrm{N}$, and $\mathrm{Ni}$ vacancy complexes. The formation energy calculation confirms $\mathrm{Ni}_{S}$ as a more stable state compared to $\mathrm{Ni}_{I}$, consistently with previous studies.$^{28,30}$ More interestingly, it shows that a nickel pair $\mathrm{Ni}_{S} \mathrm{Ni}_{S}$ yields a $2.7 \mathrm{eV}$ lower energy than two separated $\mathrm{Ni}_{S}$ atoms, suggesting a tendency for 


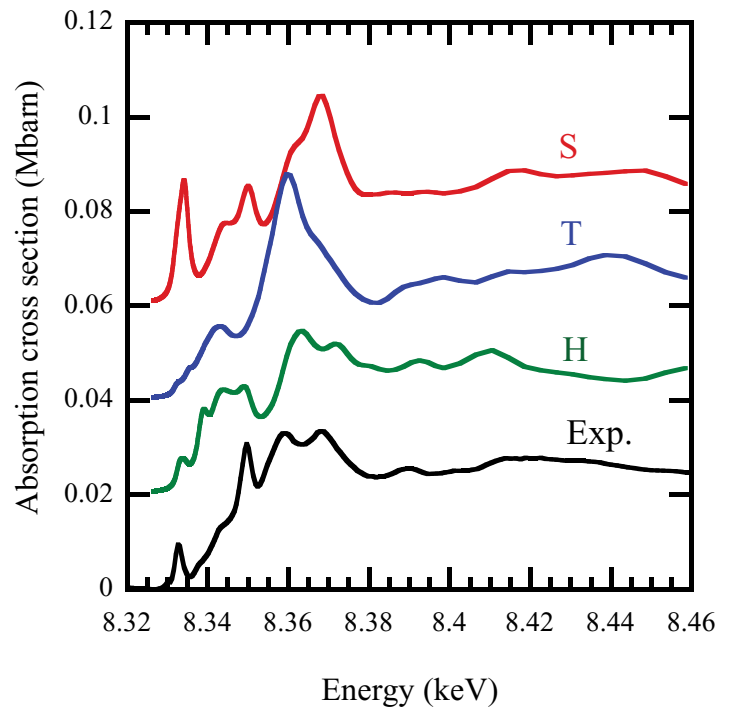

FIG. 8. (Color online) Experimental XANES and FDMNES simulated XANES spectra for substitutional $\mathrm{Ni}_{S}$ and interstitial $\mathrm{Ni}_{I}$ sites, $\mathrm{H}$ corresponds to the hexagonal site and $\mathrm{T}$ to the tetrahedral one. Spectra have been shifted vertically for clarity. Atomic positions used for the simulations are the relaxed ones.

cluster formations. In the sample studied, however, magnetic measurement indicates no ferromagnetic behavior associated with nickel clusters, but a clear paramagnetic signature related to isolated, noninteracting magnetic sites. The formation of nickel clusters was therefore prevented either by growth kinetics or by the incorporation of isolated nickel in a configuration even more energetically favorable than the cluster. With the nickel divacancy having a total energy $1.7 \mathrm{eV}$ lower than that of the substitutional $\mathrm{Ni}_{S}$, it is the most favorable configuration.

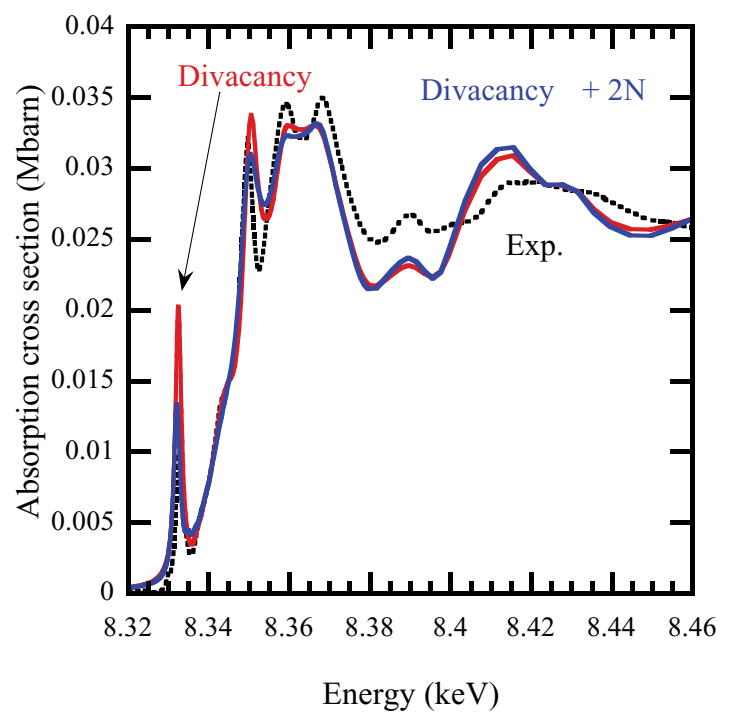

FIG. 9. (Color online) Experimental XANES and FDMNES simulated XANES spectra for Ni divacancy lattice sites. Model with and without $\mathrm{N}$ atoms are shown. They are very similar. The divacancy model is better than the substitutional $\mathrm{Ni}_{S}$ and interstitial $\mathrm{Ni}_{I}$ site models. Atom positions are not relaxed.
Total energies cannot be compared directly because of different chemical compositions, but a diamond crystal containing two isolated $\mathrm{Ni}_{S}$ atoms will gain $2.7 \mathrm{eV}$ when forming a nickel pair and $3.4 \mathrm{eV}$ when forming two isolated divacancies. Thus, the calculations suggest that nickel is more favorably incorporated on divacancy sites in diamond.

From an experimental point of view, the XANES spectrum combined with FDMNES simulations shows clearly that nickel was not incorporated in either the substitutional site or the interstitial site. The only agreement between this experiment and the simulations is obtained for the nickeldivacancy complex. Minor variations are observed on the simulated spectra depending on the number and positions of nitrogen atoms decorating the divacancy, but no clear conclusion can be drawn. Most probably the sample contains a mixture of divacancies decorated with zero to four nitrogen atoms.

These results should be compared with those of magnetooptical studies of the same crystal at low temperature and high field, ${ }^{39}$ which showed that the $\mathrm{Ni}$ main luminescence line at $1.4 \mathrm{eV}$ had its trigonal axis along the [111] direction in the (111) growth sectors and was distributed among the four equivalent [111] directions in the (100) growth sectors. According to a recent study, ${ }^{30}$ the only Ni-divacancy complexes with a trigonal symmetry are those which are not decorated with any nitrogen atom, and this only in the neutral or -2 charge and $1 / 2$ spin states. Although a general picture where $\mathrm{Ni}$ is incorporated on divacancy sites would seem compatible with the features of this $1.4-\mathrm{eV}$ line, a more detailed study of the effect of nitrogen on the electronic structure and charge state of such a defect is necessary before making a more affirmative assignment. Complementary measurements on the same crystal such as x-ray circular magnetic dichroism (XCMD) on the $\mathrm{Ni} \mathrm{L}_{2,3}$ edge could provide information on the magnetic moment of the divacancy centers.

\section{CONCLUSION}

The present work reports a theoretical and experimental investigation of $\mathrm{Ni}$ and related defects in diamond. DFT calculations were performed for different possible structures and computed results are compared to the XANES results. Theoretical calculations and experimental investigations suggest that a divacancy lattice site is most stable for nickel in the studied diamond crystal.

\section{ACKNOWLEDGMENTS}

We thankfully acknowledge P. Gaikwad (ENS Cachan) for help during the experiment at the ESRF. A.K. is thankful to the Agence Nationale pour la Recherche (Grant No. ANR 06 BLAN 0339-02), France, for providing financial support during this research. We are also thankful to J. F Roch and F. Treussart (LPQM, ENS Cachan) for fruitful discussions. The XCRYSDEN program was used to plot the relaxed atomic positions. $^{52}$ 
*Etienne.Gheeraert@ grenoble.cnrs.fr

${ }^{\dagger}$ Present address: Institute for Experimental and Applied Physics, University of Regensburg, D-93040 Regensburg, Germany.

${ }^{\ddagger}$ Present address: Tsukuba Expo Center, Japan.

${ }^{1}$ A. Gruber, A. Drabenstedt, C. Tietz, L. Fleury, J. Wrachtrup, and C. von Borczyskowski, Science 276, 2012 (1997).

${ }^{2}$ A. Lohrmann, S. Pezzagna, I. Dobrinets, P. Spinicelli, V. Jacques, J. F. Roch, J. Meijer, and A. M. Zaitsev, Appl. Phys. Lett. 99, 251106 (2011).

${ }^{3}$ J. R. Maze, P. L. Stanwix, J. S. Hodges, S. Hong, J. M. Taylor, P. Cappellaro, L. Jiang, M. V. G. Dutt, E. Togan, A. S. Zibrov, A. Yacoby, R. L. Walsworth, and M. D. Lukin, Nature (London) 455, 644 (2008).

${ }^{4}$ G. Balasubramanian, I. Y. Chan, R. Kolesov, M. Al-Hmoud, J. Tisler, C. Shin, C. Kim, A. Wojcik, P. R. Hemmer, A. Krueger, T. Hanke, A. Leitenstorfer, R. Bratschitsch, F. Jelezko, and J. Wachtrup, Nature (London) 455, 648 (2008).

${ }^{5}$ C. D. Clark, H. Kanda, I. Kiflawi, and G. Sittas, Phys. Rev. B 51, 16681 (1995).

${ }^{6}$ Chunglang Wang, C. Kurtsiefer, H. Weinfurter, and B. Burchard, J. Phys. B 39, 37 (2006); U. F. S. D’Haenens-Johansson, A. M. Edmonds, B. L. Green, M. E. Newton, G. Davies, P. M. Martineau, R. U. A. Khan, and D. J. Twitchen, Phys. Rev. B 84, 245208 (2011). ${ }^{7}$ A. Collins and P. Spear, J. Phys. D Appl. Phys. 15, L183 (1982).

${ }^{8}$ S. Lawson, H. Kanda, K. Watanabe, I. Kiflawi, Y. Sato, and A. Collins, J. App. Phys. 79, 4348 (1996).

${ }^{9}$ A. M. Zaitsev, Phys. Rev. B 61, 12909 (2000).

${ }^{10}$ T. Gaebel, I. Popa, A. Gruber, M. Domhan, F. Jelezko, and J. Wrachtrup, New J. Phys. 6, 98 (2004).

${ }^{11}$ E. Wu, V. Jacques, F. Treussart, H. Zeng, P. Grangier, and J.-F. Roch, J. Lumin. 119, 19 (2006).

${ }^{12}$ I. Aharonovich, C. Zhou, A. Stacey, J. Orwa, S. Castelletto, D. Simpson, A. D. Greentree, F. Treussart, J.-F. Roch, and S. Prawer, Phys. Rev. B 79, 235316 (2009).

${ }^{13}$ S. Pezzagna, D. Rogalla, and D. Wildanger, New J. Phys. 13, 035024 (2011).

${ }^{14}$ M. Wolfer, A. Kriele, O. A. Williams, H. Obloh, C. C. Leancu, and C. E. Nebel, Phys. Status Solidi A 206, 2012 (2009).

${ }^{15}$ T. Dietl, H. Ohno, and F. Matsukura, Phys. Rev. B 63, 195205 (2001).

${ }^{16}$ S. C. Erwin and C. S. Hellberg, Phys. Rev. B 68, 245206 (2003).

${ }^{17}$ E. B. Lombardi, Diamond Relat. Mater. 17, 1345 (2008).

${ }^{18}$ J. Rabeau, Y. Chin, S. Prawer, F. Jelezko, T. Gaebel, and J. Wrachtrup, Appl. Phys. Lett. 86, 131926 (2005).

${ }^{19}$ M. Benabdesselam, P. Iacconi, D. Briand, A. Berkane-Krachai, E. Gheeraert, and H. Kanda, J. Appl. Phys. 88, 4648 (2000).

${ }^{20}$ M. Benabdesselam, P. Iacconi, E. Gheeraert, H. Kanda, D. Lapraz, and D. Briand, Radiat. Prot. Dosim. 100, 329 (2002).

${ }^{21}$ J. Maes, K. Iakoubovskii, M. Hayne, A. Stetsmans, and V. M. Moshchalkov, J. Phys. D: Appl. Phys. 37, 1102 (2004).

${ }^{22}$ L. Paslovsky and J. E. Lowther, J. Phys. Chem. Solids 54, 243 (1993).
${ }^{23}$ Y. Jinlong, Z. Manhong, and W. Kelin, Phys. Rev. B 49, 15525 (1994).

${ }^{24}$ J. E. Lowther, Phys. Rev. B 51, 91 (1995).

${ }^{25}$ J. Goss, A. Resende, R. Jones, S. Öberg, and P. R. Briddon, Mater. Sci. Forum 196, 67 (1995).

${ }^{26}$ K. Johnston and A. Mainwood, Diamond Relat. Mater. 11, 631 (2003).

${ }^{27}$ M. Watkins and A. Mainwood, J. Phys.: Condens. Matter 15, S2913 (2004).

${ }^{28}$ J. P. Goss, P. R. Briddon, R. Jones, and S. Öberg, J. Phys.: Condens. Matter 16, 4567 (2004).

${ }^{29}$ R. Larico, L. V. C. Assali, W. V. M. Machado, and J. F. Justo, Appl. Phys. Lett. 84, 720 (2004); 85, 6293 (2004).

${ }^{30}$ R. Larico, J. F. Justo, W. V. M. Machado, and L. V. C. Assali, Phys. Rev. B 79, 115202 (2009).

${ }^{31}$ J. Isoya, H. Kanda, J. R. Norris, J. Tang, and M. K. Bowman, Phys. Rev. B 41, 3905 (1990).

${ }^{32}$ J. Isoya, H. Kanda, and Y. Uchida, Phys. Rev. B 42, 9843 (1990).

${ }^{33}$ V. A. Nadolinny, A. P. Yelisseyev, J. M. Baker, M. E. Newton, D. J. Twitchen, S. C. Lawson, O. P. Yuryeva, and B. N. Feigelson, J. Phys.: Condens. Matter 11, 7357 (1999).

${ }^{34}$ K. Iakoubovskii, A. Stesmans, B. Nouwen, and G. J. Adriaenssens, Phys. Rev. B 62, 16587 (2000).

${ }^{35}$ J. M. Baker, J. Phys.: Condens. Matter 15, S2929 (2003).

${ }^{36}$ K. Iakoubovskii, Phys. Rev. B 70, 205211 (2004).

${ }^{37}$ M. H. Nazaré, A. J. Neves, and G. Davies, Phys. Rev. B 43, 14196 (1991).

${ }^{38}$ P. W. Mason, F. S. Ham, and G. D. Watkins, Phys. Rev. B 60, 5417 (1999).

${ }^{39}$ P. Plochocka, O. Portugall, P. Y. Solane, E. Gheeraert, L. Ranno, E. Bustarret, N. Bruyant, I. Breslavetz, D. K. Maude, H. Kanda, and G. L. J. A Rikken, Phys. Rev. B 86, 045203 (2012).

${ }^{40}$ G. Kresse and J. Hafner, Phys. Rev. B 47, 558 (1993).

${ }^{41}$ J. P. Perdew and Y. Wang, Phys. Rev. B 33, 8800 (1986).

${ }^{42}$ G. Kresse and J. Hafner, J. Phys.: Condens. Matter 6, 8245 (1994).

${ }^{43}$ H. J. Monkhorst and J. D. Pack, Phys. Rev. B 13, 5188 (1976).

${ }^{44}$ D. Saada, J. Adler, and R. Kalish, Phys. Rev. B 61, 10711 (2000).

${ }^{45}$ J. P. Goss, R. Jones, M. I. Heggie, C. P. Ewels, P. R. Briddon, and S. Öberg, Phys. Rev. B 65, 115207 (2002).

${ }^{46}$ Y. Joly, Phys. Rev. B 63, 125120 (2001).

${ }^{47}$ G. Davies, A. J. Neves, and M. H. Nazaré, Europhys. Lett. 9, 47 (1989).

${ }^{48}$ A. T. Collins, H. Kanda, J. Isoya, C. A. J. Ammerlaan, and J. A. van Wyk, Diamond Relat. Mater. 7, 333 (1998).

${ }^{49}$ S. C. Lawson and H. Kanda, Diamond Relat. Mater. 2, 130 (1993).

${ }^{50}$ J. P. Goss, R. Jones, S. J. Breuer, P. R. Briddon, and S. Öberg, Phys. Rev. Lett. 77, 3041 (1996).

${ }^{51}$ D. J. Twitchen, J. M. Baker, M. E. Newton, and K. Johnston, Phys. Rev. B 61, 9 (2000).

${ }^{52}$ A. Kokalj, Comput. Mater. Sci. 28, 155 (2003). 